Note

\title{
Inhibitory Effects of Allium Vegetable Extracts on Fatty Acid Synthase
}

\author{
Xue-Bing Sun and Wei-Xi TIAN ${ }^{*}$ \\ College of life science, Graduate University of Chinese Academy of Sciences, Beijing, China
}

Received October 12, 2008; Accepted February 16, 2009

\begin{abstract}
Allium vegetables have been shown to have beneficial effects against several diseases, including hyperlipidemia and cancer. This study was carried out with enzymatic method to investigate the inhibition on fatty acid synthase by nine Allium vegetables to explore their health protective mechanisms. It was found the fatty acid synthase inhibitors in most Allium vegetables extracts were concentrated in the lower polar solvents and the inhibition could be reversed by dithiothreitol. Ethyl acetate extract of red onion (Allium cepa $\mathrm{L}$., $\mathrm{IC}_{50}=0.45 \mu \mathrm{g} / \mathrm{mL}$ ) was the most active of the samples tested. In garlic, diallyl trisulfide was suggested to be an effective component which may react with the essential sulphydryl groups in the enzyme. Additionally, the Chinese chive (Allium tuberosum L.) exhibited the most total inhibitory capacity, and its inhibitory components showed more polar than that of other Allium vegetables. It is suggested that different inhibitors of FAS exist in these vegetables.
\end{abstract}

Keywords: fatty acid synthase, Allium, sulfides, enzyme inhibition

\section{Introduction}

The animal fatty acid synthase (EC 2.3.1.85, FAS) is an important enzyme in energy metabolism in vivo. It catalyzes the de novo synthesis of long chain fatty acid from acetylCoA and malonyl-CoA in the presence of NADPH (Smith, 1994). FAS function is related to many human diseases such as cancer and obesity. Human cancer cells express high levels of FAS. The inhibitors of FAS such as cerulenin, C75 and EGCG were reported to be selectively cytotoxic to human cancer cells including breast, ovary, colon, leukemia, prostate and pancreatic tumors, and they exhibited growth inhibition on some human cancers such as ovarian cancer and prostate tumor (Kuhajda, 2006; Kuhajda et al., 2000; Lyn-Cook et al.,1999). The fatty-acid synthesis as a target pathway for chemotherapy development has been identified by these studies with FAS inhibitors (Kuhajda, 2006). In addition, mice treated with cerulenin or $\mathrm{C} 75$ exhibited dramatic decreases in food intake and body weight. This action appears to be mediated by malonyl-coA that is main substrate of FAS (Loftus et al., 2000). Therefore, FAS is drawing more and more attention world-wide for its association with human diseases and it might be a possible therapeutic target for both cancer and

*To whom correspondence should be addressed.

E-mail: tianweixi@gucas.ac.cn obesity. The weight-control and anti-cancer functions of teas and tea-polyphenols incarnate the double target character of FAS. The extracts of green tea and black tea, and their components gallated catechins and theaflavin had been reported to be effective inhibitors of FAS (Du et al., 2005; Wang and Tian, 2001; Zhang et al., 2006).

Besides the well known garlic (Allium sativum L.) and onion (Allium cepa L.), several other Allium species are widely grown for culinary use, such as scallion (Allium fistulosum L.), chive (Allium schoenoprasum L.) and Chinese chive (Allium tuberosum L.). Since ancient times, these Allium vegetables have been used as common foods and for the treatment and prevention of many diseases, such as cancer, coronary heart disease, obesity, hyperlipidemia, diabetes, hypertension, cataract and disturbances of the gastrointestinal tract (Augusti, 1996). It is considered generally that the anticancer function is related to the antioxidation of the sulfide compounds in these vegetables. However, the mechanism for their controlling of lipids still keeps unclear. In this investigation, inhibitory effects of Alliums on FAS were examined. The results should be valuable to understand their health protective mechanisms and learn how they may be more appropriately utilized. 


\section{Materials and Methods}

Materials Acetyl-CoA, malonyl-CoA, NADPH, dithiothreitol (DTT) and dimethyl sulfoxide (DMSO) were purchased from Sigma. Petroleum ether, ethyl acetate, acetone and ethanol were local products of analytical grade. The nine Allium vegetables including garlic bulb, garlic bolt, garlic leaves, blanched garlic leaves, scallion, chive, Chinese chive, red onion and white onion were purchased from Beijing Chaoshifa supermarket, Yuquan Road.

Preparation of plant extracts The fresh vegetables were weighed and chopped. Then they were grinded with a high speed universal pulverizer. The grinded residues were extracted with magnetic stirrer using sequential extraction with petroleum ether, ethyl acetate, acetone and 95\% ethanol in the ratio 1:1 (w/v) for 24 hours respectively. It was done for total period of 4 days. The extracts were filtered with buchner funnel. Then the petroleum ether and ethyl acetate extracts were evaporated under reduced pressure to removed organic solvent. The residues were re-dissolved with DMSO and used in experiment. The acetone and 95\% ethanol extracts were directly used in the assay.

FAS preparation and activity assays The preparation, storage and use of FAS isolated from chicken liver were performed as described previously (Soulie et al., 1984). The final purified enzyme was determined to be homogeneous by polyacrylamide gel electrophoresis (PAGE) analysis in the presence and absence of SDS. FAS activity was determined by monitoring the decrease in absorbency at $340 \mathrm{~nm}$ resulting from the oxidation of NADPH using an Amersham Pharmacia Ultrospec 4300 pro UV-Vis spectrophotometer at $37^{\circ} \mathrm{C}$. The assay mixture contained $100 \mathrm{mM}$ potassium phosphate buffer, pH 7.0, 1 mM EDTA, $2.5 \mu \mathrm{M}$ acetyl-CoA,
$10 \mu \mathrm{M}$ malonyl-CoA, $32 \mu \mathrm{M}$ NADPH, and $10 \mu \mathrm{g}$ chicken liver FAS in a total volume of $2.0 \mathrm{~mL}$ (Tian et al., 1985).

FAS inhibition assays The fast-binding inhibition was determined by adding inhibitor to the reaction system before FAS initiated the reaction. The activity of FAS in the presence of inhibitor was designated as $\mathrm{Ai}$, and the control activity of FAS in the absence of inhibitor was designated as $\mathrm{A}_{0}$. $A_{i} / A_{0}$ was the remaining activity (R.A.) of FAS that was less than 1 for the inhibition of FAS. Generally, this inhibition is induced by non-covalent loading of inhibitor on the enzyme, which is reversible. The concentrations of inhibitor required for $50 \%$ inhibition $\left(\mathrm{IC}_{50}\right)$ were obtained from the dose-response curves of inhibition. The organic extract solvent contained in the assay mixture was not over $2.5 \%$. It had been checked that the DMSO, acetone or ethanol of less than $2.5 \%$ in the assay nearly did not affect on the activity of FAS.

The time-dependent inhibition was determined by taking aliquots to measure the remaining activity at a series of time intervals after the FAS was mixed and incubated with the inhibitor. A time course of the activity inhibition was achieved. Commonly, this inhibition is resulted by covalent reaction of inhibitor with the enzyme, so that it is irreversible.

\section{Results and Discussion}

Fast-binding inhibition on FAS by extracts of Allium As is shown in Table 1, all tested extracts of vegetables from the family Allium exhibit fast-binding inhibition on FAS in vitro. The inhibition were dose-dependent. It was found that lower polar extracts petroleum ether extracts and ethyl acetate extracts exhibited stronger inhibition than higher polar extracts did, and their $\mathrm{IC}_{50}$ values ranged between $0.45 \mu \mathrm{g} / \mathrm{mL}$

Table 1. Fast-binding inhibition of vegetable extracts from the family Allium on FAS activity.

\begin{tabular}{cccccc}
\hline \multirow{2}{*}{$\begin{array}{c}\text { Sample } \\
\text { No. }\end{array}$} & Allium vegetables and species (in bracket) & \multicolumn{2}{c}{$\mathrm{IC}_{50}$ values of different solvent extracts $(\mu \mathrm{g} / \mathrm{ml})$} \\
\cline { 3 - 6 } & $\begin{array}{c}\text { Petroleum } \\
\text { ether }\end{array}$ & $\begin{array}{c}\text { Ethyl } \\
\text { acetate }\end{array}$ & Acetone & 95\% ethanol \\
\hline 1 & Chinese chive (Allium tuberosum L.) & $8.10 \pm 0.17$ & $13.8 \pm 0.03$ & $13.6 \pm 0.12$ & $28.0 \pm 0.81$ \\
2 & Garlic bulb (Allium sativum L.) & $2.35 \pm 0.21$ & $3.39 \pm 0.14$ & $96.0 \pm 10.35$ & $353 \pm 15.40$ \\
3 & Garlic bolt (Allium sativum L.) & $2.88 \pm 0.19$ & $4.15 \pm 0.25$ & $247 \pm 0.87$ & $171 \pm 4.17$ \\
4 & Garlic leaves (Allium sativum L.) & $3.25 \pm 0.16$ & $5.82 \pm 0.47$ & $321 \pm 27.93$ & $83.7 \pm 2.06$ \\
5 & Blanched garlic leaves (Allium sativum L.) & $1.70 \pm 0.48$ & $4.84 \pm 0.41$ & $138 \pm 2.47$ & $101 \pm 3.67$ \\
6 & Red onion (Allium cepa L.) & $1.71 \pm 0.02$ & $0.45 \pm 0.02$ & $225 \pm 3.66$ & $108 \pm 8.67$ \\
7 & White onion (Allium cepa L.) & $5.40 \pm 0.34$ & $1.27 \pm 0.06$ & $117 \pm 1.87$ & $61.1 \pm 2.28$ \\
8 & Scallion (Allium fistulosum L.) & $1.80 \pm 0.13$ & $3.44 \pm 0.59$ & $311 \pm 2.20$ & $123 \pm 11.02$ \\
9 & Chive (Allium schoenoprasum L.) & $9.38 \pm 0.45$ & $9.00 \pm 0.32$ & $228 \pm 31.61$ & $58.3 \pm 0.55$ \\
\hline
\end{tabular}

The $\mathrm{IC}_{50}$ values were mean \pm standard deviation $(n=3)$ 
and $13.8 \mu \mathrm{g} / \mathrm{mL}$. There were some other interesting nuances between these extracts. Four garlic samples and scallion showed their strongest inhibitory activity in the petroleum ether extracts, whereas two onion samples displayed the better inhibition in the ethyl acetate extracts. The Chinese chive, however, did not show much difference among the extracts of these four organic solvents. This result indicated that there are some different inhibitors in the Allium plants.

The most potent inhibitor was the ethyl acetate extract of red onion whose $\mathrm{IC}_{50}$ values was $0.45 \mu \mathrm{g} / \mathrm{mL}$. Though these inhibitors are extracts rather than pure compounds, their inhibitory abilities are much greater than that of the previously reported FAS inhibitors, such as cerulenin whose $\mathrm{IC}_{50}$ values was $20 \mu \mathrm{g} / \mathrm{mL}$ (Vance et al., 1972), EGCG whose $\mathrm{IC}_{50}$ values was $24 \mu \mathrm{g} / \mathrm{mL}$ (Wang and Tian, 2001).

Time-dependent inhibition of FAS by extracts of Allium Petroleum ether extracts re-dissolved with DMSO from 9 Allium samples were respectively mixed with FAS solutions to make a final concentration of $2 \mu \mathrm{g} / \mathrm{mL}$ extracts. The relative activities of FAS were determined at different incubation time. The proportion of DMSO in the mixtures was $4 \%$. Little effect of gradually 1-5\% DMSO on the activity of FAS under the same condition had been previously observed. It was found that all 9 petroleum ether extracts have time-dependent inhibition on FAS. Fig. 1A showed the progressive inhibition of petroleum ether extracts from garlic bulb which had the fastest and strongest impact among these samples.

$40 \mathrm{mM}$ of DTT were added to FAS reaction mixtures that had been completely inactivated by $10 \mu \mathrm{g} / \mathrm{mL}$ of these petroleum ether extracts, and activities of the enzyme was continuously assayed for 90 minutes. It was observed that the activities of the FAS all gradually recovered over time following the addition of DTT (Fig 1B).

The result of DTT reactivation indicated that the components of Allium plants should interact with the essential sulphydryl groups of FAS.

Total volume of inhibitory capacity on FAS in the extracts of Allium Defining the inhibitory capacity of FAS inhibitor to be one unit which inhibits $50 \%$ FAS activity in $1 \mathrm{~mL}$ assay solution on the basis of fresh weight of the vegetables, it was found that the Chinese chive contains the most of total inhibitory capacity. The total inhibitory unit percentages of extracts of these nine vegetables by four solvents are shown in Fig. 2, while the total unit percentage of Chinese chive was $100 \%$.

The results exhibited that the most inhibitory activity of Chinese chive was concentrated in the extract by acetone which has higher polar among these four solvents. The most FAS inhibitors in garlic were extracted by non-polar solvent petroleum ether. The over $50 \%$ of inhibitory activity in onion dissolved in ethyl acetate which polar is intervenient between acetone and petroleum ether. The results suggested that there was some difference for the FAS inhibitors in different Allium vegetables. It is assumed that nearly non-polar diallyl trisulfide is the major FAS inhibitor in garlic, the main inhibitory component in onion is di(1-propenyl) trisulfide. It has been reported that many sulfides are preventive or therapeutic to various cancers and other diseases. Many experimental studies have demonstrated that diallyl disulfide, S-allylcysteine, and ajoene have inhibitory effects on carcinogenesis in animals (Le Bon and Siess, 2000; Powolny and Singh, 2008).
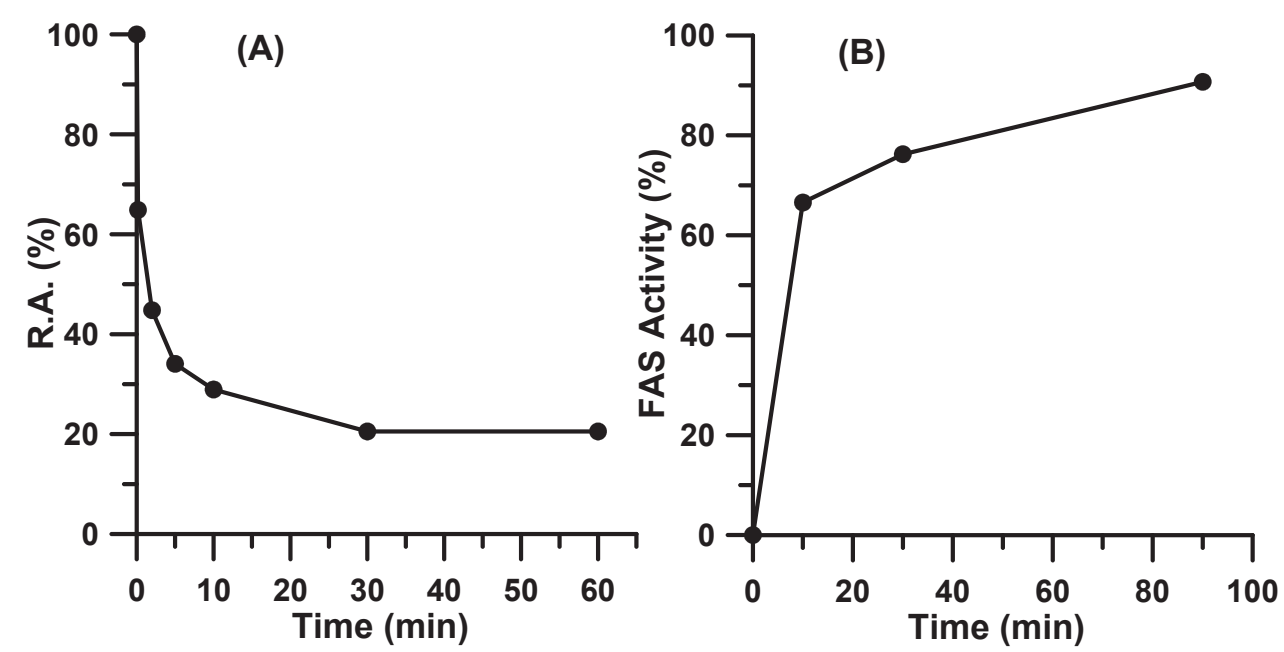

Fig. 1. Chemically reversible time-dependent inhibition of FAS by extracts of Alliums. (A) Inhibition course of petroleum ether extract from garlic bulb. The concentration of FAS in the inactivation system was 0.824 $\mu \mathrm{M}$ and the concentration of garlic bulb extract was $2 \mu \mathrm{g} / \mathrm{mL}$. (B) Reactivation of FAS by DTT. FAS had been totally inactivated by $10 \mu \mathrm{g} / \mathrm{mL}$ petroleum ether extracts of garlic bulb previously. Reactions contained $0.824 \mu \mathrm{M}$ FAS. The concentration of DTT in the mixtures was $40 \mathrm{mM}$. 


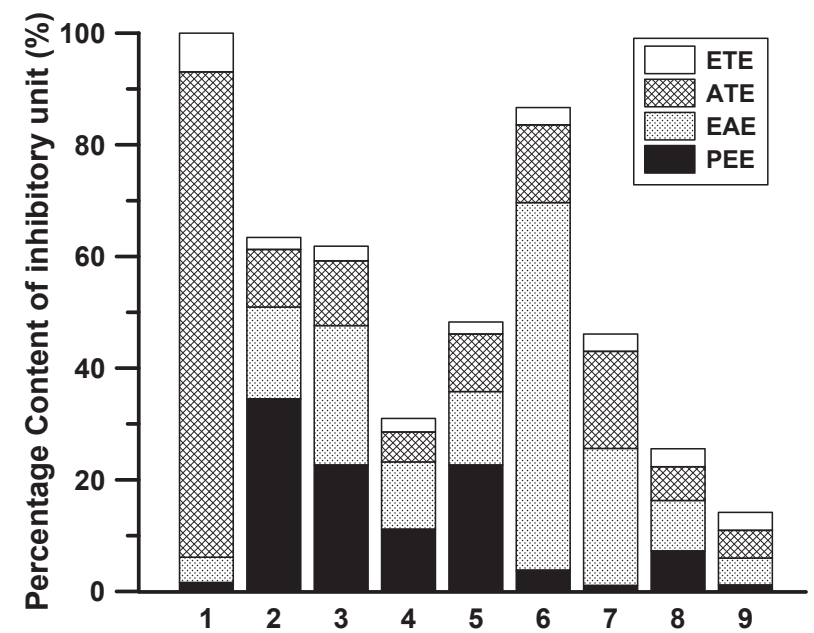

Fig. 2. Total inhibitory unit percentages of extracts of nine Allium vegetables. The total unit percentage of Chinese chive was $100 \%$, 1. Chinese chive, 2. garlic bulb, 3. garlic bolt, 4. Garlic leaves, 5. blanched garlic leaves, 6. red onion, 7. white onion, 8. scallion, 9. chive. ETE, 95\% ethanol extracts; ATE, acetone extracts; EAE, ethyl acetate extracts; PEE, petroleum ether extracts.

In Chinese chive, the polar of most inhibitory components is higher than the trisulfide. However, the most polar solvent ethanol extracted the least inhibitors. It is shown that the FAS inhibitors in Alliums were different from the known natural FAS inhibitors, such as tea-polyphenols and flavonoids (Tian, 2006).

In this study, the results showed that the extracts of Alliums strongly inhibited FAS and the mechanism might be related to the interaction with essential sulphydryl groups of FAS. In addition, different inhibitors could exist in various Allium plants. The results of our study provided a new point of view to explore the health protective mechanisms of Allium plants.

Acknowledgements This work was supported by Grants 30572252 and 30670455 of the National Natural Science Foundation of China.

\section{References}

Augusti, K. T. (1996). Therapeutic values of onion (Allium cepa L.) and garlic (Allium sativum L.). Indian J. Exp. Biol., 34, 634-640.

Du, Y. T., Wang, X., Wu, X. D. and Tian, W. X. (2005). Keemun black tea extract contains potent fatty acid synthase inhibitor and reduces food intake and body weight of rats via oral intubation. $J$. Enzyme Inhib. Med. Chem., 20, 349-356.
Kuhajda, F. P. (2006) Fatty acid synthase and cancer: new application of an old pathway. Cancer Res., 66, 5977-5980.

Kuhajda, F. P., Pizer, E. S., Li, J. N., Mani, N. S., Frehywot, G. L. and Townsend, C. A. (2000). Synthesis and antitumor activity of an inhibitor of fatty acid synthase. Proc. Natl. Acad. Sci. U. S. A., 97, 3450-3454.

Le Bon, A. M. and Siess, M. H. (2000). Organosulfur compounds from Allium and the chemoprevention of cancer. Drug Metabol. Drug Interact., 17, 51-79.

Loftus, T. M., Jaworsky, D.E., Frehywot, G. L., Townsend, C. A., Ronnett, G. V., Lane, M. D. and Kuhajda, F. P. (2000). Reduced food intake and body weight in mice treated with fatty acid synthase inhibitors. Science, 288, 2379-2381.

Lyn-Cook, B. D., Rogers, T., Yan, Y., Blann, E. B., Kadlubar, F. F. and Hammons, G. J. (1999). Chemopreventive effects of tea extracts and various components on human pancreatic and prostate tumor cells in vitro. Nutr. Cancer, 35, 80-86.

Powolny, A. A. and Singh, S. V. (2008). Multitargeted prevention and therapy of cancer by diallyl trisulfide and related Allium vegetable-derived organosulfur compounds. Cancer Lett., 269,305-314.

Smith, S. (1994). The animal fatty acid synthase: one gene, one polypeptide, seven enzymes. Faseb. J., 8, 1248-1259.

Soulie, J. M., Sheplock, G. J., Tian, W. X. and Hsu, R. Y. (1984). Transient kinetic studies of fatty acid synthetase. A kinetic selfediting mechanism for the loading of acetyl and malonyl residues and the role of coenzyme A. J. Biol. Chem., 259, 134-140.

Tian, W. X. (2006). Inhibition of fatty acid synthase by polyphenols. Curr. Med. Chem., 13, 967-977.

Tian, W. X., Hsu, R. Y. and Wang, Y. S. (1985). Studies on the reactivity of the essential sulfhydryl groups as a conformational probe for the fatty acid synthetase of chicken liver. Inactivation by 5,5' -dithiobis-(2-nitrobenzoic acid) and intersubunit cross-linking of the inactivated enzyme. J. Biol. Chem., 260, 11375-11387.

Vance, D., Goldberg, I., Mitsuhashi, O. and Bloch, K. (1972). Inhibition of fatty acid synthetases by the antibiotic cerulenin. Biochem. Biophys. Res. Commun., 48, 649-656.

Wang, X. and Tian, W. (2001). Green tea epigallocatechin gallate: a natural inhibitor of fatty-acid synthase. Biochem. Biophys. Res. Commun., 288, 1200-1206.

Zhang, R., Xiao, W. P., Wang, X. D., Wu, X. and Tian, W. X. (2006). Novel Inhibitors of fatty acid synthase from green tea (Camellia sinensis Xihu Longjing) with high activity and new reacting site. Biotechnol. Appl. Biochem., 43, 1-7. 